\title{
The differential effects of conditioned stimulus intensity and chlorpromazine on avoidance
}

HARRY L. CHIPMAN

PURDUE UNIVERSITY

The effects of different levels of an auditory conditioned stimulus on the acquisition of an avoidance response with and without chlorpromazine pretreatment were examined. Ss trained under the highest intensity made more avoidance responses than other groups. All pretreated groups were below their respective controls. Results indicate that $S_{S}$ trained under conditions of greater sensory stimulation will learn faster and that chlorpromazine can attenuate this effect.

One of the outstanding features of the reticular formation is its relation to the primary sensory pathways. Lying in the core of the brainstem, the reticular formation receives many collaterals from these tracts. These connections form a part of the ascending reticular system which has a major role in the arousal process.

In his proposal of a sensory drive Isaac (1960) discussed the functional relationship of the primary sensory paths to the reticular formation. He demonstrated that sensory stimulation can have an effect on reticular formation activity similar to that of direct electrical stimulation. It is accepted that increased reticular formation activity produces behavioral arousal. Further, the aroused or attentive animal is more likely to solve a problem than the non-attentive one (Galambos \& Morgan, 1960). Taken together these conclusions suggest that an animal trained under conditions of increased sensory stimulation should learn faster.

The experiment reported here proposed to test the hypothesis above. The studies of Isaac and others have not employed aversive stimuli. However, a two-way shuttlebox avoidance paradigm was chosen as the experimental task. An auditory conditioned stimulus provided the experimentally controlled sensory stimulation. Much of the work on which the hypothesis under test is based was done in cats. However, the albino rat was chosen as the experimental animal. Therefore, the hypothesis was tested in a different experimental setting on a different species.

If the reticular formation was involved in the sensory stimulation effect, attenuating it would impair acquisition. A number of traditional physiological procedures could have been used to produce the attenuation. However, their usage would have placed constraints on the interpretation of results. A pharmacologic procedure, chlorpromazine pretreatment, was used to test the assumption of reticular formation involvement. Bradley (1963) concluded that chlorpromazine neither depresses the reticular formation or the primary sensory pathways nor does it have any systematic effect in the unanesthetized, intact animal. However, its action appears to be selectively related to the influence of afferent impulses impinging on the reticular formation via collaterals from the primary sensory tracts. Therefore, chlorpromazine would produce the desired attenuation. Methods and Procedure

Twenty-four adult male albino rats of the PurdueWistar strain were used as Ss. They were randomly assigned in groups of four to treatment combinations of a 3 by 2 factorial design, three levels of conditioned stimulus intensity against either a drug or saline control condition. The three levels of the auditory conditioned stimulus used were: 84,74 , and $64 \mathrm{~dB}$. Ss assigned to drug groups received $2 \mathrm{mg} / \mathrm{kg}$ chlorpromazine $30 \mathrm{~min}$. prior to each testing session. All others received a placebo injection of saline.

The apparatus was a two-compartment shuttlebox with an electrifiable grid floor. The compartments were separated by a partition which had a cut-out centered at floor level forming a passageway between the two. A two-way avoidance conditioning paradigm using foot shock was the experimental task. Each animal was given 15 conditioning trials per day for four consecutive days.

Results

The mean number of avoidance responses was computed for each treatment combination (see Fig. 1).

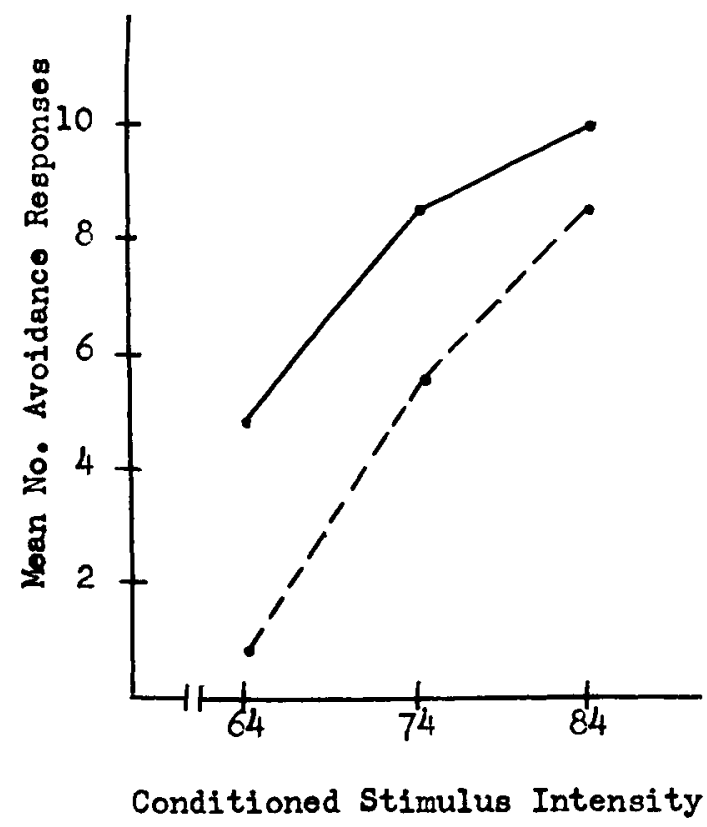

Fig. 1. Changes in mean number of avoidance responses as a function of conditioned stimulus intensity for rats injected with chlorpromazine $2 \mathrm{mg} / \mathrm{kg}$ (dashed line) or with .6 ml saline (solid line). 
Differences among these were assessed by a nonparametric test for differences in central tendency, the Randomization Test for Two Independent Samples (Siegel, 1956). Significant differences $(p<.05)$ were found between controls at the extreme conditioned stimulus intensities and between drug and control groups at the lowest intensity. A difference $(p<.10)$ existed between the drug groups at the extreme conditioned stimulus intensities.

\section{Discussion}

The data for the no-drug control groups indicate that the number of avoidance responses increased with increases in conditioned stimulus intensity. A similar relationship appeared to exist for the drug groups. However, the drug effect could not be distinguished from the conditioned stimulus intensity effect at the upper two intensities. This was not a parametric study and the exact shape of the function relating these variables is not known.

The outcome of the experiment reported here supports the hypothesis previously offered and is in agreement with Isaac's (1960) findings in the cat. Further, drug treated Ss made fewer avoidance responses than the respective nontreated control Ss. In view of chlorpromazine's action, the assumption of reticular formation involvement in the conditioned stimulus intensity effect would appear tenable. In general, the results of this experiment are in agreement with current theory of reticular formation function.

\section{References}

Bradley, P. B. Phenothiazine derivations. In W. S. Root \& F. G. Hofman (Eds.), Physiological pharmacology. New York: Academic Press, 1963. Pp. 417-447.

Galambos, R., \& Morgan, C. T. The neural basis of learning. In J. Field (Ed.), Handbook of Physiology. Sect. 1. Neurophysiology. Vol. III. Baltimore: Williams \& Wilkins, 1960. Pp. 1471-1499. Isaac, W. Arousal and reaction times in cats. J. comp. physiol. Psychol., 1960, 53, 234-236.

Siegel, S. Nonparametric statistics for the behavioral sciences. New York: McGraw-Hill, 1956. 